Mit Verdruß sah Muhammed Ali auf seinen vielen Besichtigungsfahrten, wieviel Arbeitskraft dem Lande verlorenging durch die pharaonische Methode der Bewässerung mittels des Schwengelschöpfers (Schaduf), Göpelbrunnens (Sakkiye) oder der archimedischen Wasserschnecke.

Muhammed Alis Traum war eine systematische Bewässerung des Deltas, bei möglichst gleichbleibendem Wasserspiegel. Mougel Bey, der französische Ratgeber Muhammed Alis, legte dem deutschen Besucher bereits die fertigen Pläne vor. Das Wehr sollte unfern der Stelle errichtet werden, wo sich der Nil, der im Altertum sieben, heute nur noch zwei Mündungsarme aufweist, in seine Arme gabelt. Man hat das Dellta zutreffend mit einem ausgebreiteten Fächer verglichen. Was beim Fächer der kostbare Knauf ist, sollte am grünen Riesenfächer des Deltas der Staudamm werden: ein Wahrzeichen Ägyptens, Symbol des Lebens.

1843 wurde mit der Ausführung begonnen. Der Damm zeigte mehrere Jahre nach seiner Vollendung kleine Mängel - ein Schicksal, das er mit dem von den Engländern erbauten Assuanstaudamm teilte - und die Erhöhung des Assuandammes machte abermals neue Eingriffe erforderlich.

Griechen erwarben sich in den folgenden Jahrzehnten große Verdienste um die ägyptische Baumwollkultur, Schweizer um ihre Weiterverarbeitung zu Gespinsten höchster Qualität - aber den Grund zur Blüte hat Muhammed Ali gelegt durch seinen «Barrage du Nil», den der Leser im beigegebenen Farbbild schaut, welches dem bei Kümmerly \& Frey in Bern erschienenen Ägypten-Buch des Verfassers entnommen ist.

\title{
ON THE CULTURAL GEOGRAPHY OF THE NILE DELTA
}

In the course of history, function and importance of the Nile Delta region has changed several times. It was known for its resorts and hunting grounds during the time of the Pharaos. In the course of the Islamic era it developed into a cotton-producing area. Farming became more intensive after construction of a dam for irrigation in the middle of the 19th century. This intensification has been a steady trend in the agriculture of the Delta ever since.

\section{DIE CHINESEN IN VIETNAM}

\section{WERnER KüNDig-Steiner}

Seit Jahrtausenden wandern die Chinesen übers Meer nach Süden. Die Hauptanziehungspunkte sind die Deltatiefländer Südostasiens und des Malayischen Archipels. In manchen Ländern dieses Raumes bilden die Chinesen die bedeutendste Minoritätengruppe. Dieser «Chinesen-Mangel» hat südlich des 650-Millionen-Blockes überrascht. Ein Hauptgrund liegt darin, daß sich die auswandernden Chinesen in den südlicheren, tropisch-subtropischen Tiefebenen Südostasiens viel leichter niederlassen konnten als in dem von indomongoliden Stämmen besetzten, kontinentalgebirgigeren, zum Teil savannen- und steppenhaften Nordabschnitt. Von den gesamthaft etwa 11 Millionen zählenden Auslandchinesen beherbergt Südvietnam rund 800000 oder 7\%, Nordvietnam $0,5 \%$. Es soll im Folgenden gezeigt werden, wann und wo sich ihre Einwanderung vollzog und in welcher Art sich heute die Chinesen ins Wirtschaftsleben Vietnams einzuschalten vermochten.

Die Chinesenauswanderung nach Vietnam begann bereits im 3. und 4. Jahrhundert und verstärkte sich später spürbar bis ums Jahr 10oo. Diese älteren Auswanderergruppen verschmolzen restlos mit den ansäßigen Annamiten. Davon zeugen die Familiennamen der Wong, Lee, Chen, Hu oder Tang. Auch im spätern Mittelalter spiegelt die Zuwanderung das Bild der innern Zustände Chinas. In Zeiten sozialer Unsicherheiten stieg der Zustrom von Flüchtlingen,

Legende zum Bild rechts: Nildelta mit dem Stauwerk Barrage du Nil 


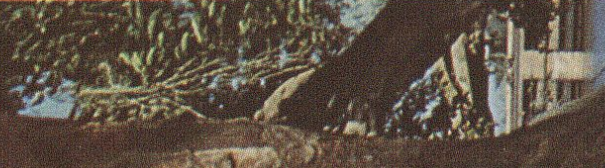

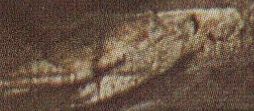

19x

Whather

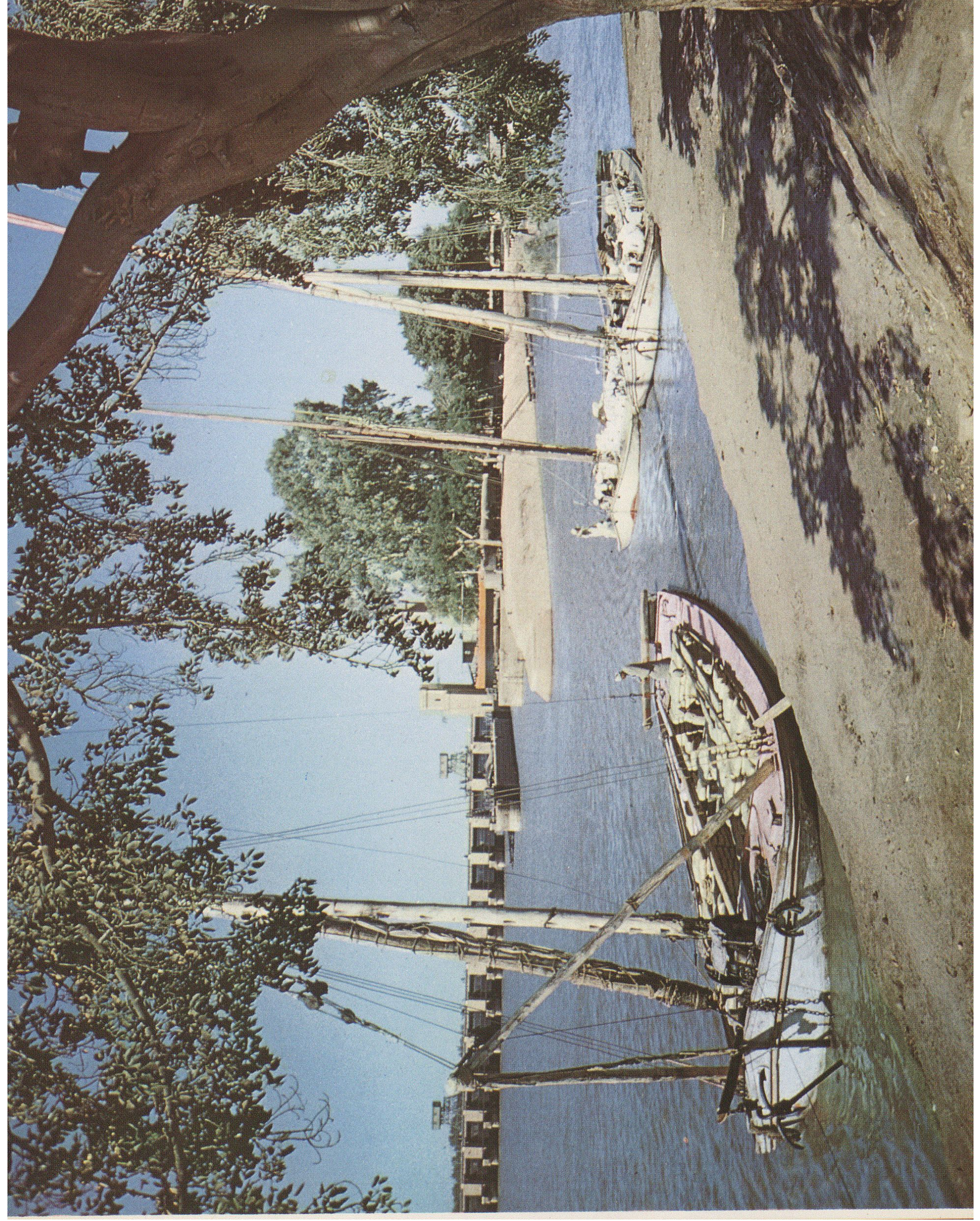




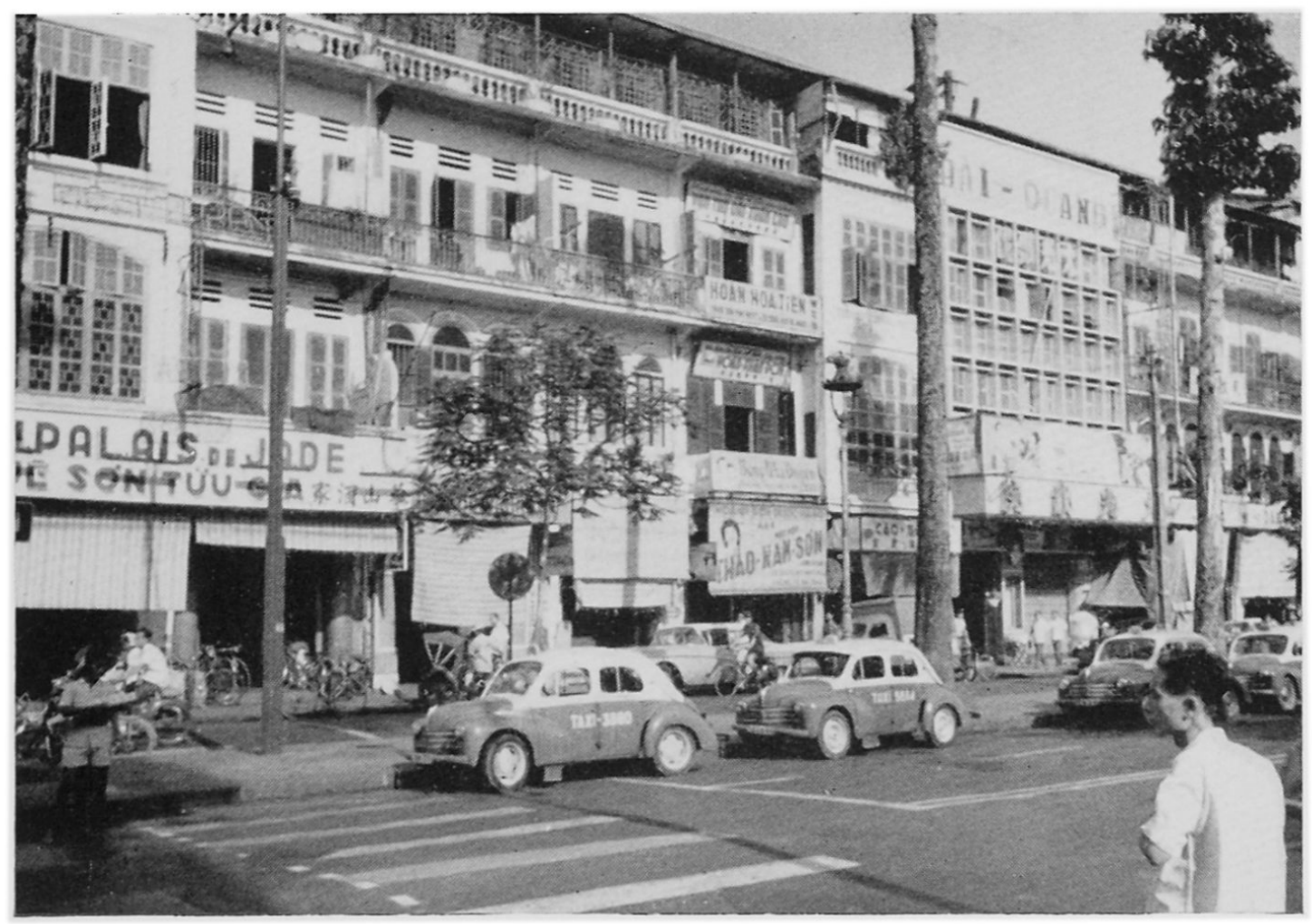

Cholon. Stadtzentrum mit Kaufläden, Büros, Wohnungen und Hinterhöfen. Photo: W. Kündig-Steiner

in friedlicheren Zeiten ging er zurück. Spätmittelalterliche Auswandererfamiliennamen sind jene der Miao, der Man und der Lolo; ihre verwandtschaftlichen Beziehungen reichen heute noch nach Zentralchina. Quellenmäßig verbürgt sind stärkere Zuwanderungen im 17. Jahrhundert. Die Leute siedelten sich als Holzfäller und Ackerbauer bei Bien Hoa, to km östlich der Stadt Saigon, an und legten damit den Grundstock zu einer typischen Chinesenregion Südvietnams. Die friedliche Infiltration setzt sich auch im 18. und 19. Jahrhundert fort, d.h. solange die Oberherrschaft Chinas über Annam nicht durch die französische Kolonisierung (1858-19o7) gebrochen war. Noch vor der Jahrhundertwende gingen die direkten chinesischen Kultureinflüsse spürbar zurück; um so mehr entwickelte sich unter dem Schilde der Kolonialregierung der wirtschaftliche Einfluß der Auslandchinesen. Mehr als je erscheinen auf dem Dorfe jene Händler und Krämer, die, sehr strebsam und sparsam, mehr und mehr den $Z$ wischenhandel beherrschen. Zur Erschließung Indochinas wünschten sich die französischen Kolonialbehörden vor allem chines.sche Straßen- und Eisenbahnbauarbeiter, auch Hilfskräfte für die Plantagenwirtschaft. Aber 1890 verzeichnet Vietnam erst um etwa 60000 Chinesen, somit erst rund $1 / 12$ der heutigen Zahl.

Der zur andernde Kontinentalchinese bevorzugte das zentrale Mekongdelta. In diesem in der $Z$ wische, kriegszeit stark sinisierten Raum entstanden nicht nur neue wirtschaftliche Kleinzentren, sordern gleichzeitig auch jene religösen Sekten und Geheimbünde, die besonders der jungen vietnamischen Regierung zu schaffen machten. Vor wenigen Jahren erst wurden diese politisch-militärischen Gemeinschaften mit Waffengewalt zerschlagen. Heute noch deckt sich der engere Wir!. Ingsraum dieser chinesischen Bünde mit den latenten Rebellenkampfgebieten.

Mit der Zweiteilung Vietnams im Jahre 1954 kamen im Flüchtlingsstrom von 1,2 Millionen etwa 45000 Chinesen aus Norden mit, die sich größtenteils in der Region der großen Chinesenstadt Cholon bei Saigon niederließen. Cholon, das heute rund 3/4 Millionen zählt, vermochte diesen Zustrom sehr gut zu assimilieren. Es beherbergt heute gut $1 / 2$ Million Chinesen, somit um $2 / 3$ aller. Jeder Fremdling kann in Cholon in rein chinesischer Atmosphäre leben.

Die wirtschaftliche Aktivität der Chinesen in Vietnam ist heute augenfällig. In Landwirtschaft, Industrie, Handel und Bankwesen, aber auch in den sozialen Diensten und in der Schulung sind sie pionierhaft. Wenn im kleinsten Dorf ein Kramladen eröffnet wird, so gehört er bestimmt einem Chinesen, der von hier aus zunächst in eine Kleinstadt und später einmal in den Raum Cholon-Saigon übersiedelt. Fast alle Gründungen von Tee- und Pfefferplantagen beruhen auf initativen Chinesen. Die Grüngemüsepflanzungen rund um Saigon gehören meistens Chinesen. Unternehmungs- und 
Arbeitswillen zeigen auch die 1954/55 aus Nordvietnam bzw. aus dem Delta des Roten Flusses um Hanoi zugeströmten Gärtner, die einst aus den klassischen Gemüsebauprovinzen von Kanton und Fou-Kien auswanderten. In manchen Provinzen, wie etwa in Ba-Xuyen und An-Xuyen (d.h. im küstennahen Mekongdelta), begannen die Chinesen mit dem Reisbau, verließen ihn und übernahmen den Reistransport zur Mühle und auch zum Exportschiff. Im weitern sind in den Holzregionen Südvietnams viele Sägewerke in chinesischer Hand. Sehr oft begannen die Betriebseigentümer als Arbeiter z.B. einer Tabak- oder Gummiplantage, um dann sehr langsam zum Unternehmer emporzusteigen. Schon seit Ende des 19. Jahrhunderts entwickelte sich die Raffinage und die Vermahlung des Reises zu einer typisch chinesischen Industrie. Die erste große Reisschälerei Vietnams baute 1878 ein Chinese. Heute gibt es in Cholon etwa 70 Reisschälereien, die alle in chinesischer Hand sind und täglich 8-9000 t Reis verarbeiten können. Mehr und mehr folgt auch der chinesische Unternehmer der Tendenz, sich multi-industriell zu beschäftigen, wenn möglich Produkte zu schaffen, die ins übrige Asien exportiert werden können. Die Chinesen besitzen die meisten Zuckerraffinerien Vietnams, auch die meisten Betriebe zur Verarbeitung von Kokos- und Erdnüssen, für chemische Produkte wie Seife und Dünger. Charakteristisch für den großen chinesischen Anteil im Transportwesen sind die Schiffswerften, deren erste vor gut 100 Jahren eine chinesische war. 1927 gab es in Vietnam etwa 30 chinesische Schiffsbaufirmen. Heute sind es noch deren 10; aber unter ihnen befindet sich die größte des Landes. Sie hat sich auf den Bau von Flußschiffen für den Reistransport spezialisiert. Endlich sei auf die Textilfabriken hingewiesen, die sich fast ausschließlich in Cholon befinden. Es sind familiäre Werkstätten, in denen gleichzeitig Seide, Baumwolle und neuerdings auch Kunstfasern verarbeitet werden. Im weitern sind die Chinesen in der Porzellanindustrie, in der Gummi-, der Kerzen- und der Weihrauchherstellung die führenden Fabrikanten des Landes.

Am meisten überrascht, daß die großen chinesischen Banken relativ spät errichtet wurden. Erst 1946 wurden die jungen Zweigstellen der Hongkonger «East-AsiaBank» von Hanoi und Haipong nach Saigon verlagert und ein Jahr später nach Cholon ausgedehnt. Bereits 1927 legte die chinesische Verkehrsbank eine Filiale in Saigon und eine in Cholon an. Die älteste Vertretung - seit 1910 - besitzt die chinesische Handelskammer in Saigon, deren Geschäftsleiter bis 1959 stets Glieder eines Stammverbandes, d. h. eines Clans sein mußten. In bestimmter Reihenfolge lösten sich diese Direktoren aus dem Mutterlande ab. Schon 1949 wurde - auf dem Papier wenigstens - diese von außen kommende Clanaufsicht abgeschafft.

Dies alles vermag zu zeigen, daß der chinesische Einflu $\beta$ in Vietnam, insbesondere wirtschaftlich, nicht unterschätzt werden darf. Dies gilt nicht zuletzt auch für die zirka 1400 vietnamischen Import-Export-Firmen, von denen aber nur 250 chinesische sind. Aber diese kleinere Gruppe ist ebenso aktiv wie die 50 chinesischen Handelsgesellschaften, in denen nochmals $1350 \mathrm{H}$ andelsfirmen zusammengeschlossen sind. Wohl einen der wichtigsten Zusammenschlüsse bildet der Verband der ohinesischen Mediziner, auch der Juweliere, nicht zu vergessen die Teehausbesitzer und die Konfektionäre. In mehreren modernen Wirtschaftsverbänden besitzen die Chinesen die absolute Mehrheit.

Nicht nur für die vietnamesischen, sondern für alle Auslandchinesen ist bezeichnend, daß sie großes Gewicht auf eine systematische Schulung ihrer Jugend legen. Wer in Vietnam höhere Schulen besuchen wollte, mußte bis um die Jahrhundertwende nach Hongkong, Kanton oder Schanghai fahren. Erst um 1908 waren die Chinesen von Cholon so weit, daß sie - mit Bewilligung der Franzosen - ein Handelslyzeum eröffnen konnten. Um 1930 gab es in Vietnam rund 125 chinesische Schulen, 1960 waren es 230, wovon fast die Hälfte im Raume Saigon-Cholon. Auch heute noch gehören die Quartierschulen für Chinesen privaten Institutionen; die höhern, meist öffentlich 


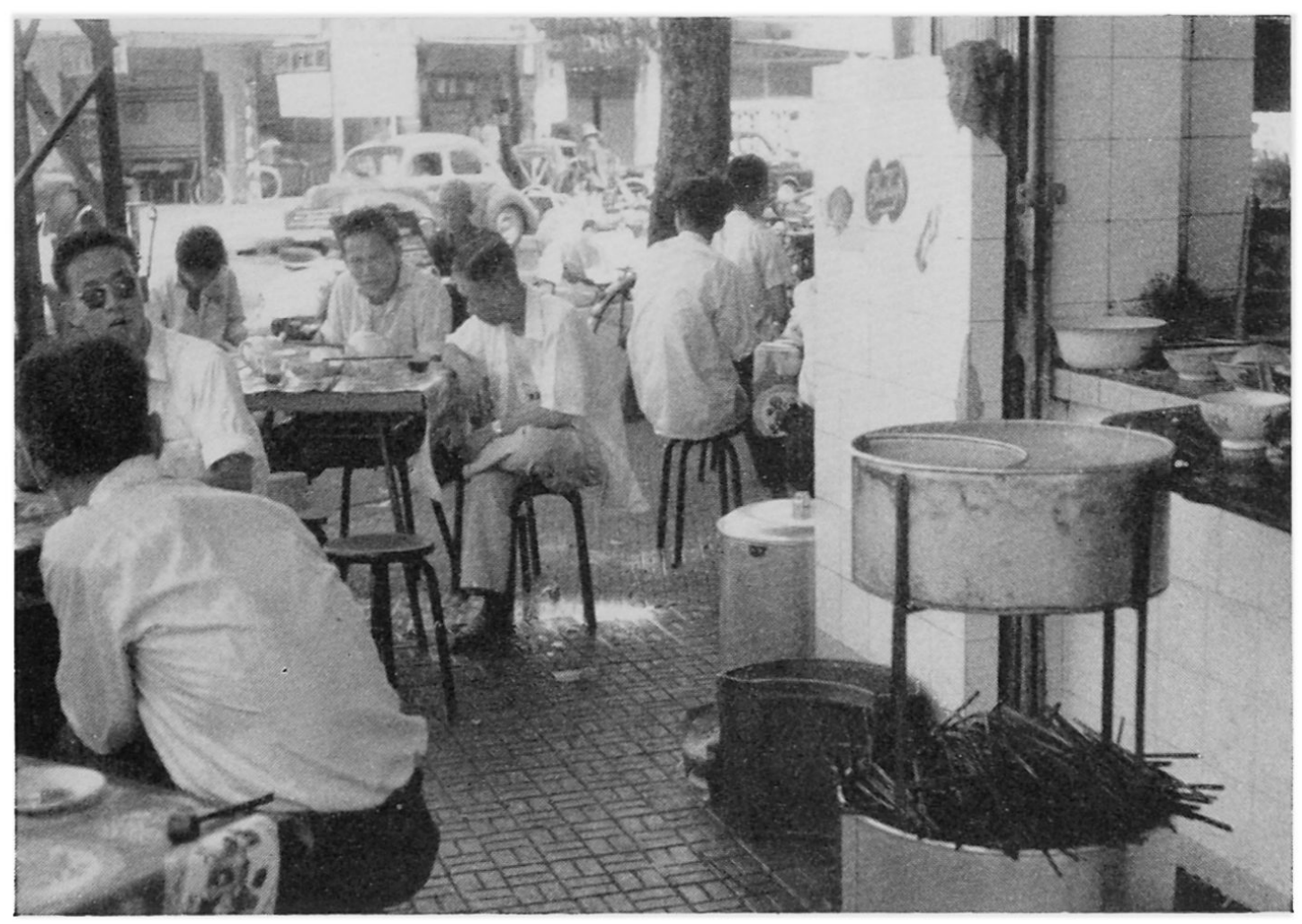

Saigon. Chinesisches Quartierrestaurant. Photo: W. Kündig-Steiner

unterstützten Schulen werden sehr oft von einer Stammverwandtschaft, d.h. von einem Clan, geleitet, der ganz bestimmte Lehrziele verfolgt, die mit denen der jungen Republik Vietnam nicht gänzlich übereinstimmen. Seit kurzem wird auch in den chinesischen Schulen Vietnamesisch als Unterrichtssprache verlangt. Man versucht durchwegs, die Chinesen zu guten vietnamesischen Staatsbürgern zu erziehen. - In diesem Zusammenhang sei auch die chinesische Lokalpresse erwähnt: das sind 11 militant antikommunistische Zeitungen, die sämtliche in Cholon erscheinen und auch die Hauptstädte von Kambodscha und Laos erreichen. Erwähnenswert sind auch die besondern Leistungen der Chinesen im Gesundheitsdienst. Er läßt sich durchaus mit westlichen Verhältnissen vergleichen. Die fünf größten Spitäler - alle im Raume Saigon-Cholon - gehören chinesischen Organisationen und werden meist wiederum von Clans geleitet. Es scheint, daß sich die chinesischen Spitäler heute mehr als zuvor auch der vietnamesischen Bevölkerung öffnen. Dies alles bedeutet, daß sich die 800000 Auslandchinesen Vietnams zunehmend zu vietnamesischen Staatsbürgern umformen. Schon immer stellte sich heraus, daß der vietnamische Chinese leichter zu assimilieren war als etwa sein Bruder in Malaya. In erster Linie springt der Chinese in die Lücken auswandernder Franzosen (wie in Vietnam) oder Engländer (wie in Malaya). In der Tat schreitet die Sinisierung Südostasiens nicht nur bevölkerungsmäßig, sondern vor allem wirtschaftlich ungehindert vorwärts. Der Tag scheint nicht mehr fern zu sein, an dem nach der wirtschaftlichen Eroberung dieses Raumes durch die chinesische Intelligenz auch die politische Durchdringung viel systematischer als gegenwärtig einsetzen wird.

\section{THE CHINESE IN VIETNAM}

The Chinese in Vietnam, numbering about 1 million, have done pioneer work in several segments of Vietnamese economy, e.g. in agriculture, manufacturing industries, and trade. They also are very active in the cultural life of the country; Chinese culture virtually dominates the Vietnamese urban scenery, but cultural and economic influence of the Chinese is penetrating the whole country. 